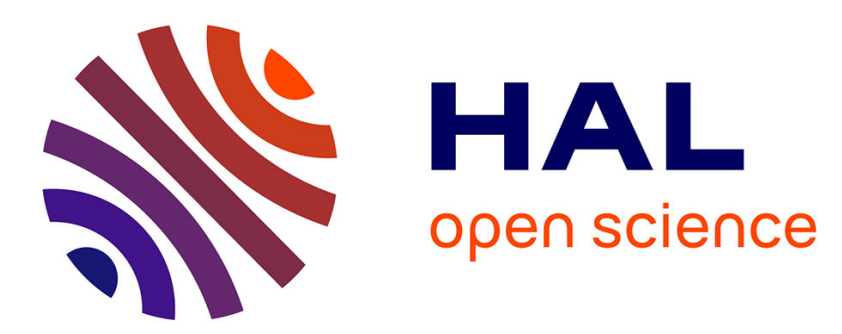

\title{
A Comparative Study Between Cubic and Ellipsoidal Fokker-Planck Kinetic Models
}

Eunji Jun, Marcel Pfeiffer, Luc Mieussens, M. Hossein Hossein Gorji

\section{To cite this version:}

Eunji Jun, Marcel Pfeiffer, Luc Mieussens, M. Hossein Hossein Gorji. A Comparative Study Between Cubic and Ellipsoidal Fokker-Planck Kinetic Models. AIAA Journal, 2019, 57 (6), pp.2524-2533. 10.2514/1.J057935 . hal-02506158

\section{HAL Id: hal-02506158 \\ https://hal.science/hal-02506158}

Submitted on 12 Mar 2020

HAL is a multi-disciplinary open access archive for the deposit and dissemination of scientific research documents, whether they are published or not. The documents may come from teaching and research institutions in France or abroad, or from public or private research centers.
L'archive ouverte pluridisciplinaire HAL, est destinée au dépôt et à la diffusion de documents scientifiques de niveau recherche, publiés ou non, émanant des établissements d'enseignement et de recherche français ou étrangers, des laboratoires publics ou privés. 


\title{
A Comparative Study Between Cubic and Ellipsoidal Fokker-Planck Kinetic Models
}

\author{
Eunji Jun \\ German Aerospace Center (DLR), 37073 Göttingen, Germany \\ Marcel Pfeiffer \\ Institute of Space Systems, University of Stuttgart, Pfaffenwaldring 29, D-70569 Stuttgart, Germany \\ Luc Mieussens \\ Bordeaux INP, Univ. Bordeaux, CNRS, INRIA, IMB, UMR 5251, F-33400 Talence, France \\ M. Hossein Gorji \\ Computational Mathematics and Simulation Science, Mathematics Department, EPF Lausanne, Swizerland
}

\begin{abstract}
Motivated by improving the performance of particle based Monte-Carlo simulations in the transitional regime, Fokker-Planck kinetic models have been devised and studied as approximations of the Boltzmann collision operator. By generalizing the linear drift model, the cubic Fokker-Planck (cubic-FP) and ellipsoidal Fokker-Planck (ES-FP) have been proposed, in order to obtain the correct Prandtl number of $2 / 3$ for a dilute monatomic gas. This study provides a close comparison between both models in low Mach and supersonic settings. While direct simulation Monte-Carlo (DSMC) here serves as the benchmark, overall close performance between cubic-FP, ES-FP and DSMC are observed. Furthermore, while the ES-FP out-performes the cubic-FP model in the shock region of the supersonic flow around a cylinder, the latter shows a better accuracy in the near continuum regime. It is argued that the reason behind these discrepancies lies in the entropy law besides the transport properties.
\end{abstract}

\section{Nomenclature}

$$
\begin{aligned}
\mathcal{F} & =\text { distribution function } \\
K n & =\text { Knudsen number } \\
L & =\text { characteristic length, } \mathrm{m} \\
\mathbf{M} & =\text { random variable associated with velocity } \\
p & =\text { pressure, } \mathrm{Pa} \\
t & =\text { time, } \mathrm{s} \\
T & =\text { temperature, } \mathrm{K} \\
U & =\text { bulk velocity, } \mathrm{m} / \mathrm{s}
\end{aligned}
$$




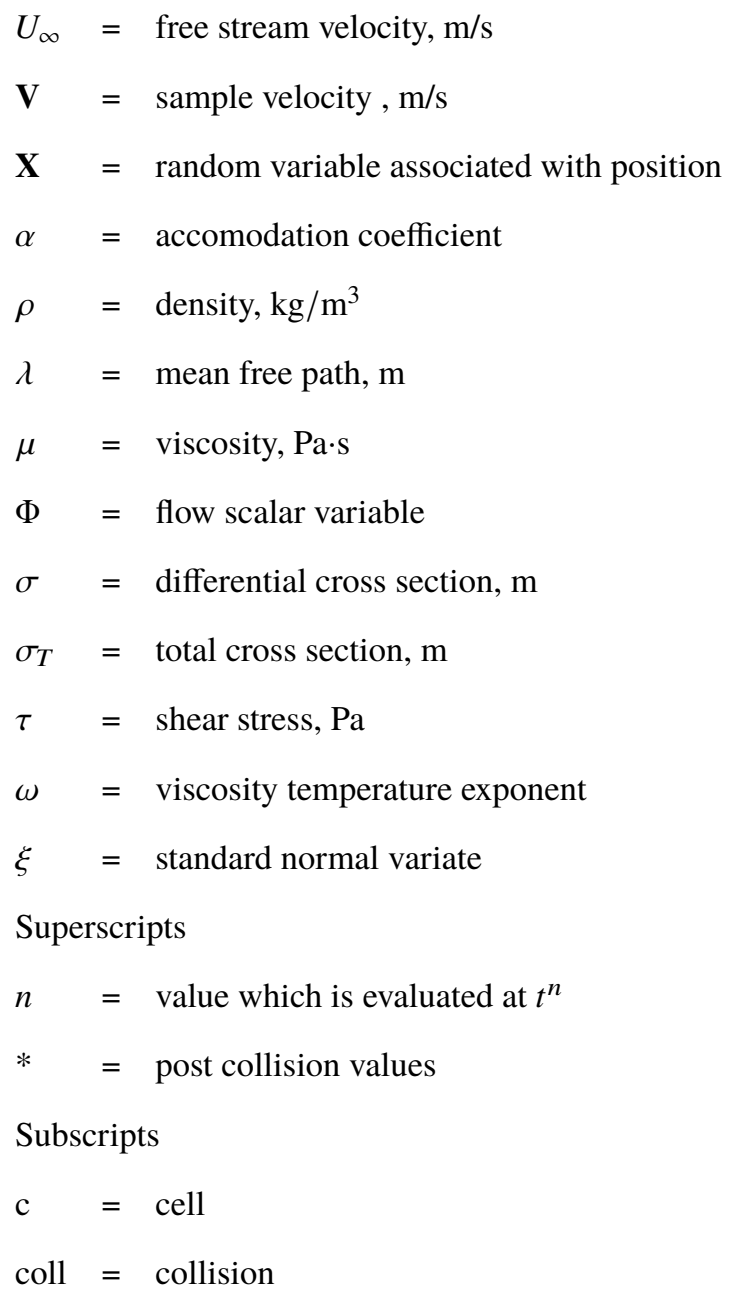

\section{Introduction}

$\mathrm{F}$ Low phenomena in rarefied regimes often lead to breakdown of conventional hydrodynamics laws. In particular the physics of gas flows subject to large Knudsen numbers which may arise in micro-nano geometrical scales or reentry maneuvers can deviate significantly from the Navier-Stokes-Fourier (NSF) description. In order to circumvent the closure assumptions underlying the NSF system, the notion of the molecular velocity distribution is relevant and necessary. The Boltzmann equation then provides an accurate governing equation for the evolution of the distribution function in the setting of dilute monatomic gases. While physically accurate description of the gas can be obtained for the whole Knudsen number range, the computational complexity associated with the Boltzmann equation motives alternative numerical schemes or even approximate physical models.

Among possible numerical methods addressing simulation of gas flows based on the Boltzmann equation, DSMC approach is one of the most advanced and widely utilized ones. In fact, DSMC employs the notion of computational 
particles whose evolution mimics translation and collision steps equivalent to the Boltzmann equation [1]. It can be shown that a converged DSMC solution is consistent with the solution of the Boltzmann equation [2]. It is important to note that over its half a century evolution, DSMC has turned into a mature solution algorithm for rarefied gas flow simulations which can accurately cope with complex physical settings, including chemical reactions, internal degrees of freedom as well as mixtures (see e.g. [3-6]).

However two fundamental constraints can result to a poor computational efficiency of DSMC based simulations. Since a stochastic version of the particle collisions are performed during each DSMC time step, the spatio-temporal discretization should honour the collisional scales, in an average sense. Hence a time step size smaller than the local mean collision time, and a cell size with a characteristic length below the local mean free path have to be chosen [7]. Therefore, DSMC becomes quite stiff in flow scenarios where some portions of the flow experience locally small Knudsen numbers. The second constraint comes from the Monte-Carlo nature of DSMC [8]. In general, the statistical error associated with Monet-Carlo sampling of a certain macroscopic quantity, scales inversely with the square root of number of particles, and proportional to the variance of the correponding microscopic variable. Therefore DSMC in its conventional form, is subject to a large statistical error in e.g. low Mach conditions.

In order to address the near-continuum issue of DSMC, a Fokker-Planck approximation of the Boltzmann collision operator has been employed in the solution algorithm devised by Jenny et al. [9]. In fact, their approximation leads to the Langevin process for describing the effect of discrete collisions among particles. This transformation of jump process to an approximate diffusive one, leads to a significant computational gain. Since here the computational particles evolve along continuous stochastic paths which are independent from each-other. Hence time steps and cell sizes larger than the corresponding collisional ones can be utilized. However note that because in the Langevin description considered in [9], only one time scale exists, a wrong Prandtl number of 3/2 is reproduced. In order to cope with that, a polynomial expansion of the drift coefficient was considered in the follow up studies by Gorji et al. [10], leading to the cubic FP model. More recently, motivated by the ellipsoidal BGK model, Mathiaud \& Mieussens proposed an anisotropic diffusion coefficient to devise a correct Prandtl number of 2/3 for the Fokker-Planck model [11]. While both cubic-FP and ES-FP models lead to an Itô diffusion stochastic processes with correct transport properties in the hydrodynamic limit, they differ in the structure of drift and diffusion coefficients and hence their performance should be assessed with respect to each-other in some representative flow setups. This is the main theme of the present article which includes the following contents.

First, some modeling aspects of the Fokker-Planck approximations are reviewed. Their theoretical differences in terms of the entropy law and moment relaxation rates are discussed. Next in $\$[1]$, an outline of the numerical schemes 
for particle Monte-Carlo simulations based on cubic-FP and ES-FP is presented. Section IV provides the problem settings and model assumptions required for the simulations. In $\$ \mathrm{~V}$, numerical experiments for assessment of both models are carried out. First, the results are shown for low Mach planar Couette and Fourier flows, where both models perform close to the benchmark DSMC. Next, high Mach Couette flow simulations are conducted. Here compared to ES-FP, a better agreement between cubic-FP and DSMC can be found in the near hydrodynamic regime which is linked to the Prandtl number issue. Finally, the supersonic flow around a cylinder is studied. Here while DSMC and ES-FP results show a close agreement in the shock region, a slight deviation of cubic-FP can be observed. It is discussed that the main reason behind the deviation between two FP models, lies in the entropy law. The paper concludes with an outlook for future works on development of more advanced FP based approximations.

\section{Review of Fokker-Planck Approximations}

For a monatomic gas, the statistical state of the gas is fully determined by the distribution function $\mathcal{F}(\mathbf{V}, \mathbf{x}, \mathbf{t})$ which is proportional to the probability density $f(\mathbf{V} ; \mathbf{x}, \mathbf{t})$ of finding a particle with a velocity close to $\mathbf{V}$, at position $\mathbf{x}$ and time $t$. By convention, $\mathcal{F}=\rho f$ is considered with the gas density $\rho=m n$, molecular mass $m$ and the number density $n$. In the dilute limit, the Boltzmann equation provides the evolution of the velocity distribution according to

$$
\frac{\mathrm{D} \mathcal{F}}{\mathrm{D} t}=\overbrace{\frac{1}{m} \int_{R^{3}} \int_{0}^{4 \pi}\left(\mathcal{F}\left(\mathbf{V}^{*}\right) \mathcal{F}\left(\mathbf{V}_{1}^{*}\right)-\mathcal{F}(\mathbf{V}) \mathcal{F}\left(\mathbf{V}_{1}\right)\right) g \sigma(\theta, g) \mathrm{d} \theta \mathrm{d}^{3} \mathbf{V}_{\mathbf{1}}}^{S^{\text {Boltz }}(\mathcal{F})}
$$

with $\mathrm{D}(\ldots) / \mathrm{D} t=\partial(\ldots) / \partial \mathrm{t}+V_{i} \partial(\ldots) / \partial x_{i}+G_{i} \partial(\ldots) / \partial V_{i}$. Here the velocity pair $\left(\mathbf{V}_{1}^{*}, \mathbf{V}^{*}\right)$ is the post collision state of the pair $\left(\mathbf{V}_{1}, \mathbf{V}\right), \sigma$ is the differential cross section of the collision, $\theta$ the solid angle which provides the orientation of the post collision relative velocity vector and $g=\left|\mathbf{V}-\mathbf{V}_{1}\right|$. Furthermore, $\mathbf{G}$ represents any external force normalized by the molecular mass (see e.g. [12]).

In a normalized setting, it is easy to see that the right hand side of Eq.(1) i.e. the Boltzmann collision operator, becomes stiff for a small Knudsen number. As a result, numerical schemes based on the stochastic process underlying the Boltzmann operator may become prohibitevly expensive in the hydrodynamic limit. However the structure of the collision operator allows for approximations which can overcome this stiffness. First of all, note that since no memory effect is present in the collision operator, the process described by the Boltzmann equation is a Markov process. Moreover, since relaxation of all moments are continuous in time, a time continuous approximation of the collision operator becomes attractive. The generic form of the continuous Markovian stochastic processes can be cast into the 
Fokker-Planck equation

$$
S^{F P}(\mathcal{F})=\left(\frac{\partial \mathcal{F}}{\partial t}\right)_{F P}=-\frac{\partial}{\partial V_{i}}\left(A_{i} \mathcal{F}\right)+\frac{1}{2} \frac{\partial^{2}}{\partial V_{i} \partial V_{j}}\left(D_{i j} \mathcal{F}\right)
$$

where the drift coefficient $\mathbf{A}$ and positive-definite diffusion $\mathbf{D}$, may depend on $\mathbf{V}$ and moments of $\mathcal{F}$. Notice that here and henceforth, the Einstein summation convention is adopted.

Given the Fokker-Planck structure [2], the modeling task is to devise $\mathbf{A}$ and $\mathbf{D}$, to ensure some consistency between the Fokker-Planck and the Botlzmann collision operator, in a sense that for a set of polynomial weights $\Psi_{\alpha} \in\left\{1, V_{i}, V_{i} V_{j}, V_{i} V_{j} V_{k}, \ldots, V_{i_{1}} \ldots V_{i_{n}}\right\}$, the FP operator honours

$$
\int_{\mathbb{R}^{n}} S^{F P}(\mathcal{F}) \Psi_{\alpha} d^{3} \mathbf{V}=\int_{\mathbb{R}^{n}} S^{\text {Boltz }}(\mathcal{F}) \Psi_{\alpha} d^{3} \mathbf{V}
$$

These consistencies normally include relaxation rates of non-equilibrium moments, besides conservation laws.

\section{A. Linear Drift}

The simplest relevant closure for the drift and diffusion, corresponds to the Langevin equation [9]

$$
\begin{aligned}
A_{i} & =-\frac{1}{\tau}\left(V_{i}-U_{i}\right) \quad \text { and } \\
D_{i j} & =\frac{\theta}{\tau} \delta_{i j}
\end{aligned}
$$

where $\mathbf{U}$ is the bulk velocity and $\theta=k T / m$, with $T$ the temperature and $k$ the Boltzmann constant. The time scale $\tau$ can be set such that correct viscosity is obtained in the hydrodynamic limit. For a Maxwell type interaction law this time scale then reads

$$
\tau=\frac{2 \mu}{p}
$$

Here $\mu$ denotes the viscosity and $p$ the ideal gas pressure. Note that the given closure results in consistency between the two operators for moments associated with the weights $\psi \in\left\{1, V_{i}, V_{i} V_{j}\right\}$. Hence mass, momentum, energy and stresses evolve consistently. Furthermore, it is important to notice that the Fokker-Planck operator with the closures (4)-(5), admits the H-theorem for the functional

$$
H(f)=\int_{\mathbb{R}^{3}} f \log f d^{3} \mathbf{V},
$$


see e.g. [13]. The rigouress form of the linear drift model comes with the price of inaccurate relaxation of the heat fluxes and hence wrong Prandtl number of $3 / 2$, which motivates the following generalizations.

\section{B. Cubic Drift}

Intuitively, stochastic forces exerted on a sample particle can be decomposed into a systematic part as the drift and a pure random counterpart controlled by the diffusion. Hence it is natural to consider an expansion of the drift force with respect to the sample variable $\mathbf{V}$. Considering e.g. an expansion with respect to the Hermite basis results in a polynomial series of the form

$$
A_{i}=c_{i}^{(0)}+c_{i j}^{(1)} v_{j}^{\prime}+c_{i j k}^{(2)} v_{j}^{\prime} v_{k}^{\prime}+\ldots
$$

with $v_{i}^{\prime}=V_{i}-U_{i}$. Now by truncating this series with as many coefficients as required for matching the moments with weights $\psi \in\left\{1, V_{i}, V_{i} V_{j}, V_{i} V_{j} V_{j}\right\}$, leads us to the cubic drift model [10]

$$
A_{i}=c_{i j}^{(1)} v_{j}^{\prime}+c_{i}^{(2)}\left(v_{j}^{\prime} v_{j}^{\prime}-3 \theta\right)-\epsilon^{2}\left(v_{i}^{\prime} v_{j}^{\prime} v_{j}^{\prime}-\frac{2 q_{i}}{\rho}\right)
$$

where $\mathbf{q}$ is the heat flux. Here the macroscopic coefficients $c_{i j}^{(1)}$ and $c_{i}^{(2)}$ are found from a system of $9 \times 9$ linear equations corresponding to the consistency between the Fokker-Planck and the Boltzmann operator for stresses and heat fluxes (see [10] for details). Moreover, the inclusion of the cubic stabilizing term is necessary to prevent the solution blow-up in finite time [14]. Note that the diffusion remains the same as Eq. (5) with the time scale given by Eq. (6).

The cubic FP model can be also extended for polyatomic gases by extending the stochastic processes for internal degrees of freedom [15]. Yet while the idea of drift expansion provides the freedom of controling relaxation of moments as many as needed, it is not possible to prove an $\mathrm{H}$-theorem for a general case. This provides a motivation to come up with an alternative approach which considers anistropic diffusion coefficient.

\section{Anisotropic Diffusion}

The main idea behind the anistropic diffusion model comes from the fact that the diffusion tensor is responsible to assign the equilibrium distribution to the particles, i.e. similar to the role of equilibrium distirbution in the BGK type models [16]. Now in the linear drift model, i.e. closures (4) and (5), this assignment happens too abrupt without considering the anistopy of the pressure tensor, resulting in a too large Prandtl number of $3 / 2$. Thus by generalizing the diffusion in a fashion similar to ES-BGK, some non-equilibrium part of the distribution can be taken into account. In particular, 
suppose

$$
P_{i j}=\int_{\mathbb{R}^{3}} v_{i}^{\prime} v_{j}^{\prime} d^{3} \mathbf{V}
$$

denotes the pressure tensor and $\lambda_{\max }$ its largest eigenvalue. Now let

$$
v=\max \left(-\frac{5}{4},-\frac{\theta}{\lambda_{\max }-\theta}\right) .
$$

Therefore, the diffusion coefficient in the ES-FP model reads

$$
D_{i j}=(1-v) \theta \delta_{i j}+v P_{i j}
$$

and the drift remains similar to the Langevin model, with the modifed time scale

$$
\tau=\frac{2(1-v) \mu}{p} .
$$

Three points have be to be noticed here. First, the ES-FP model leads to correct relaxation of moments for the weights $\psi \in\left\{1, V_{i}, V_{i} V_{j}\right\}$ independent of $\lambda_{\max }$. Second, while the presented closure leads to the correct Prandtl number of $2 / 3$ in the Chapmann-Enskog hydrodynamic limit, the relaxation rate of the heat fluxes may not be correct (depending on $\left.\lambda_{\max }\right)$. And finally, the ES-FP model admits the H-theorem [11]. Also notice that in the follow up study, an extension to polyatomic gases with rotational energy levels was pursued [17].

\section{Numerical Methods}

The benefit of the FP type kinetic formulations lies on their continuous stochastic process structure. Let us again consider 22, it is a classic result of stochastic methods that this equation describes the distribution of particles whose space-velocity states i.e. $(\mathbf{X}(\mathbf{t}), \mathbf{M}(\mathbf{t}))$ evolve according to the system

$$
\begin{aligned}
d M_{i} & =A_{i} d t+d_{i j} d W_{j} \\
d X_{i} & =M_{i} d t,
\end{aligned}
$$

where $d_{i j} d_{j k}=D_{i j}$, and $d \mathbf{W}$ is the standard three-dimensional Wiener process [18]. Now depending on the coefficients A and $\mathbf{D}$, different time integration schemes can be devised for numerical approximation of the particle evolution. The general strategy is to assume that over each time step, the macroscopic coefficients are constant and hence only dependency on the random variable $\mathbf{M}$ would be taken into account. Through this assumption, the integration can be 
done in an exact fashion for the linear drift and ES-FP models, whereas a mixed integration scheme can be obtained for the cubic-FP model. Derivation of time integration schemes for linear-, cubic- and ES-FP models have been presented in [9], [10] and [19], respectively. Therefore here only final results are reviewed.

Let the superscripts $n$ and $n+1$ denote the approximate values provided by the scheme at time steps $t^{n}$ and $t^{n+1}=t^{n}+\Delta t$, respectively. The fluctuating part of the velocity reads $\mathbf{M}^{\prime}=\mathbf{M}-\mathbf{U}$, with $\mathbf{U}$ being the bulk velocity. Furthermore, $\xi$ is a three-dimensional standard normal random variable.

\section{A. Cubic Fokker-Planck Model}

By adopting the time integration scheme devised in [20], the velocity-position update follows

$$
\begin{aligned}
M_{i}^{n+1} & =\frac{1}{\alpha}\left(M_{i}^{\prime} e^{-\Delta t / \tau}+\left(1-e^{-\Delta t / \tau}\right) \tau N_{i}^{n}+\sqrt{\theta^{n}\left(1-e^{-2 \Delta t / \tau}\right)} \xi_{i}\right)+U_{i}^{n} \\
\text { and } X_{i}^{n+1} & =X_{i}^{n}+M_{i}^{n} \Delta t
\end{aligned}
$$

with the nonlinear contribution

$$
N_{i}=c_{i j}^{(1)} M_{j}^{\prime}+c_{i}^{(2)}\left(M_{j}^{\prime} M_{j}^{\prime}-3 \theta\right)-\epsilon^{2}\left(M_{i}^{\prime} M_{j}^{\prime} M_{j}^{\prime}-\frac{2 q_{i}}{\rho}\right)
$$

which is integrated based on the Euler-Maruyama time integration, whereas the energy conservation is fulfilled by the correction factor

$$
\alpha^{2}=1+\frac{\tau}{3 \theta}\left(\tau\left(1-e^{-\Delta t / \tau}\right)^{2}\left\langle N_{i}^{n} N_{i}^{n}\right\rangle+2\left(e^{-\Delta t / \tau}-e^{-2 \Delta t / \tau}\right)\left\langle M_{i}^{\prime n} N_{i}^{n}\right\rangle\right)
$$

with $\langle\ldots\rangle$ being the expectation. Note that the time scale $\tau$ is based on (6).

\section{B. Ellipsoidal Fokker-Planck Model}

Since the drift and diffusion coefficients for the ES-FP model are still linear with respect to M, similar to the linear drift model, an exact time integration can be devised (as carried out in [19]). Therefore the exact integration for the velocity reads

$$
M_{i}^{n+1}=M_{i}^{\prime n} e^{-\Delta t / \tau}+d_{i j}^{n} \sqrt{\left(1-e^{-2 \Delta t / \tau}\right)} \xi_{j}+U_{i}^{n}
$$

where $\mathbf{d} \mathbf{d}^{\mathbf{T}}=\mathbf{D}$, the matrix $\mathbf{D}$ given in 12 , and $\tau$ is given by (13). While a similar approach can be employed to derive an exact joint evolution of the position $\mathbf{X}$, for simplicity of the algorithm, we keep the position update similar to (17). 


\section{Resolution Requirements}

The main source of error in the described particle state updates comes through the assumption that the macroscopic coeffcients $(\mathbf{U}, \theta, \ldots)$ stay constant for a given $\Delta t$ and inside a given computational cell. It is then natural to link the spatio-temporal resolution required by the Fokker-Planck type algorithms to some variational scales of the macroscopic variables. A straight-forward approach was followed in [21], where the required spatial resolution was linked to the macroscopic gradient length scales. Let $\Phi(x, t)$ be a scalar flow variable with $\Phi^{j}$ and $\left(\partial \Phi / \partial x_{i}\right)^{j}$ as its value and gradient both estimated at the center of the computational cell $j$, respectively. Therefore the normalized length scale

$$
\Delta_{\Phi}^{j}=\left(\frac{\partial \Phi}{\partial x_{i}}\right)^{j} \frac{\Delta x_{i}^{j}}{\Phi^{j}}
$$

should fulfill a certain upper bound

$$
\max _{\Phi}\left(\Delta_{\Phi}^{(j)}\right)<\Delta_{\max }, \quad \Phi \in\{T, p, \rho,|U|\}
$$

in order to guarantee the accuracy of the FP update schemes.

\section{Physical Models and Simulation Cases}

Before presenting simulation results, in this section the values of molecular parameters besides physical conditions employed for the simulations are listed.

\section{A. Physical Models}

For all DSMC, cubic-FP and ES-FP simulations, the same physical models are employed for the argon flow with properties given in Table 1. To keep the study focused, molecular collisions of DSMC are simulated using the Maxwell molecular model with a reference temperature of $273 \mathrm{~K}$. Note that future works will consider effects of more

realistic collision models. The viscosity temperature exponent, $\omega$ is 1.0 , and reference diameter is $4.62 \times 10^{-10} \mathrm{~m}$. No-Time-Counter (NTC) scheme of Bird [1] with near-neighbor algorithm is used to select DSMC collision pairs during each time step. The diffusive surface reflection model with accommodation coefficient, $\alpha=1$ is used. All simulations are performed on SPARTA [22]. Note that while DSMC algorithm is already available on SPARTA, the cubic-FP and ES-FP solution algorithms were integrated by the authors into SPARTA.

Table 1 Physical properties of argon

\begin{tabular}{ccccccc}
\hline $\mathrm{Ar}$ & $\mathrm{T}_{0}, K$ & $\mu_{0}, k g /(m s)$ & $\omega$ & $\alpha$ & $\mathrm{m}, k g$ & $\mathrm{~d}, m$ \\
\hline Maxwell & 273 & $2.117 \mathrm{e}-5$ & 1.0 & 2.1403 & $6.63 \mathrm{e}-26$ & $4.62 \mathrm{e}-10$ \\
\hline
\end{tabular}




\section{B. Simulation Cases}

For the assessment of the cubic-FP and ES-FP methods, planar Fourier/Couette flows along with supersonic flow around a cylinder are considered.

\section{Subsonic Flow: Fourier/Couette}

Standard Fourier-Couette flows inside an infinitely long channel are investigated for low Mach assessment of the models. Hence the flow variables become only function of the spatial coordinate normal to the wall. Four different $\mathrm{Kn}=\lambda / \mathrm{L}$ are chosen, where $\mathrm{L}$ is the width of the channel and $\lambda$ is calculated based on the hard sphere model [1], with details given in Table 2. For the Couette scenario, the gas flow is driven by parallel motion of the walls. The walls are isothermal at temperature $T_{\mathrm{w}}=300 \mathrm{~K}$ and fully diffusive. The velocities are $\pm 150 \mathrm{~m} / \mathrm{s}$ for the left and the right wall, respectively. In the Fourier flow, temperature of stationary walls reads $300 \pm 50 \mathrm{~K}$ for the left and the right one, respectively. To keep the statistical error small, an average of 60,000 particles were used per computational cell, whereas $n_{c}=300$ cells are employed to capture the spatial gradients. The time step size is calculated based on a CFL criterion

$$
\Delta t=0.5 \frac{L}{n_{c} \theta_{w}}
$$

where $\theta_{w}$ is thermal speed computed for the wall condition.

Table 2 Flow conditions for Fourier/Couette setups

\begin{tabular}{cccc}
\hline$K n_{\infty}$ & density, $\mathrm{kg} / \mathrm{m}^{3}$ & number density, $/ \mathrm{m}^{3}$ & mean free path, $m$ \\
\hline $\mathbf{0 . 0 1}$ & $1.00 \mathrm{e}-3$ & $1.51 \times 10^{22}$ & $8.58 \times 10^{-5}$ \\
\hline $\mathbf{0 . 0 7}$ & $1.43 \mathrm{e}-4$ & $2.16 \times 10^{21}$ & $6.01 \times 10^{-4}$ \\
\hline $\mathbf{0 . 2}$ & $5.00 \mathrm{e}-5$ & $7.54 \times 10^{20}$ & $1.72 \times 10^{-3}$ \\
\hline $\mathbf{0 . 5}$ & $2.00 \mathrm{e}-5$ & $3.02 \times 10^{20}$ & $4.29 \times 10^{-3}$ \\
\hline
\end{tabular}

\section{Supersonic Flow I: Couette}

A more challenging scenario is realized by increasing the wall speeds of the Couette flow to $\pm 750 \mathrm{~m} / \mathrm{s}$, while keeping the walls temperatures at $T_{w}=273 \mathrm{~K}$. Three high Mach Couette simulations were performed with the number densities $n=1.29438 \times 10^{20} 1 / \mathrm{m}^{3}, n=1.29438 \times 10^{19} 1 / \mathrm{m}^{3}$ and $n=2.98261 \times 10^{18} 1 / \mathrm{m}^{3}$, resulting in $K n \approx 0.012, K n \approx 0.12$ and $K n \approx 0.5$, respectively. The width of the channel is set to $L=1 \mathrm{~m}$ and is discretized into $n_{c}=100$ computational cells. To limit the statistical and bias errors, each cell employs around 37,000 particles. Furthermore, the time step size of $\Delta t=3 \times 10^{-6} \mathrm{~s}$ was used for all three cases. 


\section{Supersonic Flow II: Cylinder}

For the high Mach assessment, flow of argon over a 12 inch diameter cylinder at Mach 2 is investigated. The free stream temperature assumed $200 \mathrm{~K}$ resulting in the free stream velocity $526.81 \mathrm{~m} / \mathrm{s}$. Surface of the cylinder is isothermal at $T_{w}=500 \mathrm{~K}$. The free stream density, number density and mean free path are given in Table 3 with Knudsen numbers calculated based on the free stream condition and the cylinder diameter, using the hard-sphere model for the mean free path. Gas-surface interaction were modeled using the Maxwell model with full momentum and energy accommodation. Fine uniform, coarse uniform and adaptive grid configurations are employed for simulations with details provided in Table 6 .

Table 3 Physical conditions of the supersonic flow around the cylinder

\begin{tabular}{cccc}
\hline$K n_{\infty}$ & density, $\mathrm{kg} / \mathrm{m}^{3}$ & number density, $/ \mathrm{m}^{3}$ & mean free path, $m$ \\
\hline $\mathbf{0 . 0 5}$ & $5.636 \times 10^{-6}$ & $8.494 \times 10^{19}$ & $1.241 \times 10^{-2}$ \\
\hline
\end{tabular}

\section{Result and Discussion}

\section{A. Results}

The simulation parameters for Fourier and Couette Flow are summarized in Table 4 Mean velocity and temperature profiles are shown in Figs 1 . 2 for DSMC, cubic-FP, and ES-FP methods. While overall close agreement between all three methods is achieved, we can observe differences between cubic-FP and ES-FP temperature profiles. In order to understand this discrepancy in the absence of statistical errors, we consider the same setup at high Mach speeds. The results for the temperature and velocity profiles of the high Mach Couette flow case are shown in Figs. 3 and

Table 4 Simulation parameters for Fourier/Couette flow

\begin{tabular}{c|ccccc}
\hline \multirow{2}{*}{ Kn } & Simulation cases & $\mathrm{N}_{\text {cell }}$ & $\mathrm{N}_{\mathrm{p}, \text { total }}$ & $\mathrm{N}_{\text {coll }}$ & $\Delta t$ \\
\hline \multirow{4}{*}{0.01} & DSMC & 300 & $1.8 \mathrm{e}+7$ & $2.89 \mathrm{e}+6$ & $5.72 \mathrm{e}-8$ \\
\cline { 2 - 6 } & cubic FP & 300 & $1.8 \mathrm{e}+7$ & - & $5.72 \mathrm{e}-8$ \\
\cline { 2 - 6 } & ES FP & 300 & $1.8 \mathrm{e}+7$ & - & $5.72 \mathrm{e}-8$ \\
\hline \multirow{4}{*}{0.07} & DSMC & 300 & $1.8 \mathrm{e}+7$ & $4.07 \mathrm{e}+5$ & $5.72 \mathrm{e}-8$ \\
\cline { 2 - 6 } & cubic FP & 300 & $1.8 \mathrm{e}+7$ & - & $5.72 \mathrm{e}-8$ \\
\cline { 2 - 6 } & ES FP & 300 & $1.8 \mathrm{e}+7$ & - & $5.72 \mathrm{e}-8$ \\
\hline \multirow{4}{*}{0.2} & DSMC & 300 & $1.8 \mathrm{e}+7$ & $1.44 \mathrm{e}+5$ & $5.72 \mathrm{e}-8$ \\
\cline { 2 - 6 } & cubic FP & 300 & $1.8 \mathrm{e}+7$ & - & $5.72 \mathrm{e}-8$ \\
\cline { 2 - 6 } & ES FP & 300 & $1.8 \mathrm{e}+7$ & - & $5.72 \mathrm{e}-8$ \\
\hline \multirow{3}{*}{0.5} & DSMC & 300 & $1.8 \mathrm{e}+7$ & $5.77 \mathrm{e}+4$ & $5.72 \mathrm{e}-8$ \\
\cline { 2 - 6 } & cubic FP & 300 & $1.8 \mathrm{e}+7$ & - & $5.72 \mathrm{e}-8$ \\
\cline { 2 - 6 } & ES FP & 300 & $1.8 \mathrm{e}+7$ & - & $5.72 \mathrm{e}-8$ \\
\hline
\end{tabular}


4 respectively. As expected, the overall agreement between the FP models and DSMC is worse compared with the low Mach case. Although the cubic-FP model agrees well with DSMC results for $K n \approx 0.012$, the deviation from DSMC for $K n \approx 0.12$ and $K n \approx 0.5$ becomes significant. By contrast, the ES-FP model shows better agreement in the temperature profile with DSMC for higher Knudsen numbers and especially at $K n \approx 0.5$. In order to further investigate the modeling errors associated with cubic-FP and ES-FP, the heat flux normal to the wall is extracted. Figure 5 shows the normal heat flux at different Knudsen numbers. Here the cubic-FP model provides better agreements with DSMC at all Knudsen numbers. The reason for under-performance of ES-FP seems to be that in the high Mach number scenario, the necessary $v=-5 / 4$ to guarantee the correct Prandtl number of $2 / 3$ is violated; due to the requirement of a positive definite diffusion matrix $D_{i j}$ as described in Eq. 11. Yet it is surprising to see that this deviation from correct Prandtl number can lead to more accurate temperature profiles while missing the corresponding heat flux.

The supersonic simulation parameters are summarized in Table 5 . Figures 6 and 7 provide comparisons between DSMC and cubic-FP/ ES-FP methods based on the Mach and temperature contours. The contours can be compared visually to verify the correspondence of the shock profile and the shock standoff distance. While overall good agreement is observed between DSMC, cubic-FP and ES-FP results; ES-FP provides a slightly more accurate shock profile compared to the cubic FP model. Also both cubic-FP and ES-FP performe less accurate in the wake region.

For a more detailed analysis, profiles are compared along the stagnation line, and on the lines inlined at $60^{\circ}$ and $120^{\circ}$ from the freestream direction. Again close agreements between all three methods is found.

Table 5 Simulation parameters for supersonic flow over a cylinder

\begin{tabular}{cccccc}
\hline Cases & Simulation cases & $\mathrm{N}_{\text {cell }}$ & $\mathrm{N}_{\mathrm{p}, \text { total }}$ & $\mathrm{N}_{\text {coll }}$ & $\Delta t$ \\
\hline A-1 & DSMC & 180,000 & $8.9 \mathrm{e}+7$ & $7.2 \mathrm{e}+6$ & $3.51 \mathrm{e}-6$ \\
\hline B-1 & cubic FP & 180,000 & $8.9 \mathrm{e}+7$ & - & $3.51 \mathrm{e}-6$ \\
\hline C-1 & ES FP & 180,000 & $8.9 \mathrm{e}+7$ & - & $3.51 \mathrm{e}-6$ \\
\hline
\end{tabular}

\section{B. Convergence analysis and computational cost}

One of the main advantages of the FP based simulations compared to DSMC is that the former needs more relaxed constraints related to the spatio-temporal resolution. This comes by the fact that the governing stochastic equations are continuous in the FP approximation. In this section, dependency of the supersonic flow around a cylinder simulation results on the spatial resolution of cubic-FP and ES-FP is investigated. Three different grid configurations i.e. fine, coarse, and adaptive mesh are set up with the corresponding simulation parameters as summarized in Table 6 . For 
both fine and coarse mesh, uniform grid configurations are employed. Profiles are compared along the stagnation line, on lines inclined at $60^{\circ}$ and $120^{\circ}$ from the free-stream direction in Figure 9 . One can see un-physically large diffusive profiles for cubic-FP in comparison to ES-FP, while using the coarse grid. This can be explained by noting that the numerical discretization applied to the latter is exact (for constant coefficients) while it is only first order for the former. Therefore in order to exploit the efficiency of the cubic-FP we have to be careful in adopting cell sizes.

The maximum normalized gradient of macroscopic values found from Eqs. 21 and 22 is analyzed for each grid resolution [23] and an adaptive mesh was therefore employed for a design of an efficient grid configuration. As described in Eq. 22. the cell refinement occurs when $\max _{\Phi}\left(\Delta_{\Phi}^{(j)}\right)$ is larger than $\Delta_{\max } . \Delta_{\max }$ is set to be 0.05 . We adopt a Cartesian hierarchical grid with on the fly cell refinement. In each refinement process, one cell is divided into 9 sub-cells. As a result of the refinement criterion, the cells near the shock and the boundary layer are refined leading to 12,000 extra cells compared to the initial coarse mesh setup. As shown in Fig.9 the results computed on the adaptive mesh are very accurate compared to the converged fine mesh profiles.

Besides the accuracy, it is necessary to identify the corresponding computational costs of the considered methods. A rough analysis shows that the cost of cubic-FP and ES-FP should be close. In order to find the coefficients of the former we require to solve a nine by nine linear system in each cell, while the latter requires the eigenvalue decomposition of the pressure tensor besides computing square root of a three by three matrix, again in each cell. Since typically we employ more than 50 particles per cell, the overhead cost of the mentioned computations is negligible. On the other hand, DSMC computational cost scales both with number of particles and collisions. For the considered supersonic test case, a rough cost measurement shows around one order of magnitude cost saving by using ES-FP/cubic-FP simulations on the adaptive mesh, in comparison to DSMC on the fine mesh. Note that as expected, the cost of three methods become very close while using fine mesh for all three. The reason is that in a fine enough resolution, the DSMC cost also scales mainly with number of particles (number of collisions per cell per time step become much fewer than number of particles). Therefore it is clear that in order to exploit the relaxed resolution constraint of the FP type approximations, one has to devise and utilize adaptive grid configurations [21, 24].

\section{Entropy Analysis}

By close inspection of shock thicknesses in Figures 6 and 7, the cubic-FP model provides a slightly thicker shock profile compared to DSMC and the ES-FP result. Since the main fundamental difference between the cubic-FP and ES-FP models lie on the entropy production which is controlled by the diffusion coefficient, in the following we further analyze the entropy production mechanism. 
Table 6 Simulation parameters

\begin{tabular}{cccccc}
\hline Cases & Simulation cases & Grid configuration & $\mathrm{N}_{\text {cell }}$ & $\mathrm{N}_{\mathrm{p}, \text { total }}$ & $\Delta t$ \\
\hline B-1 & cubic FP & Fine, Uniform & 180,000 & $8.9 \mathrm{e}+7$ & $3.51 \mathrm{e}-6$ \\
\hline C-1 & ES FP & Fine, Uniform & 180,000 & $8.9 \mathrm{e}+7$ & $3.51 \mathrm{e}-6$ \\
\hline B-2 & cubic FP & Coarse, Uniform & 5,000 & $7.6 \mathrm{e}+5$ & $1.41 \mathrm{e}-5$ \\
\hline C-2 & ES FP & Coarse, Uniform & 5,000 & $7.6 \mathrm{e}+5$ & $1.41 \mathrm{e}-5$ \\
\hline B-3 & cubic FP & Adaptive & 16,936 & $3.0 \mathrm{e}+6$ & $1.05 \mathrm{e}-5$ \\
\hline C-3 & ES FP & Adaptive & 16,776 & $3.0 \mathrm{e}+6$ & $1.05 \mathrm{e}-5$ \\
\hline
\end{tabular}

Consider the Fokker-Planck equation (2), and the entropy functional $S(f)=-H(f)$ defined based on (7). By multiplying the former with $\log (f)$, and taking integral over the velocity space we get

$$
\frac{\partial S}{\partial t}=\int_{\mathbb{R}^{3}} \frac{\partial A_{i}}{\partial V_{i}} f d^{3} \mathbf{V}+D_{i j} \overbrace{\int_{\mathbb{R}^{3}} f \frac{\partial}{\partial V_{i}} \log f \frac{\partial}{\partial V_{j}} \log f d^{3} \mathbf{V}}^{I_{i j}(f)},
$$

where $\mathbf{I}(\mathbf{f})$ is a semi-positive definite matrix known as the Fisher information. Furthermore, by approximating the drift coefficient with its linear Langevin form, the production reduces to

$$
\frac{\partial S}{\partial t} \approx-\frac{3}{\tau}+D_{i j} I_{i j}(f)
$$

From the equation above, one can clearly see the link between the diffusion coefficient $\mathbf{D}$ and the entropy production rate. Therefore in order to better understand the reason behind discrepancy between the cubic-FP and ES-FP models, Fig.10 shows $\operatorname{det}(\mathbf{D})$, in the supersonic setup. We observe that the ES-FP model produces much larger values for determinant of the diffusion coefficient, resulting in larger entropy production rate with respect to the cubic model (assuming same linear drift for both systems). Hence, we can further anticipate that the cubic FP model might fall short in honouring the H-theorem close to the shock regions. One of the solutions to fix this issue was considered in [25], where a cubic FP model consistent entropy law was proposed. However, investigating the mentioned model is beyond the scope of this study and we leave the corresponding analysis for future works. 


\section{Concluding Remarks}

Computational studies of rarefied gas flows in the transitional regime continue to be a challenging area. While DSMC has evolved to a powerful and robust methodology for such flows, its computational cost for low Knudsen flows can hinder efficient simulations for rarefied gas flows with large range of Knudsen number variations. One way to cope with this defficiency is provided by adopting the Fokker-Planck approximation of the Boltzmann equation as a stochastic particle model to fill the gap between the hydrodynamic limit and high Knudsen regime. Two main modeling ideas based on cubic-FP and ES-FP models were reviewed and further numerically studied in this work. Overall close agreements between both models together with DSMC could be observed. Yet better accuracy of the ES-FP model over the shock regions in the supersonic flow was found. On the other hand, the cubic-FP model showed a better performance in the near continuum regime, which is useful for hybrid FP-DSMC solution algorithms [26]. It is argued that the better performance of the ES-FP model in the shock region lies in the H-theorem while more accurate description of the cubic-FP model near the continuum is attributed to the correct Prandtl number. Hence as the main conclusion of this study, it turns out that both $\mathrm{H}$-theorem and recovering correct Prandtl number are crucial for accurate predictions. In future studies we further focus on investigating the role of entropy production on the performance of FP models in high Mach flows. Besdies, comparisons for realistic molecular potentials and polyatomic gases will be pursued.

\section{Acknowledgment}

The authors gratefully acknowledge the fruitful discussions with Julien Mathiaud. Hossein Gorji acknowledges the funding provided by Swiss National Science Foundation under the grant number 174060. 


\section{References}

[1] Bird, G. A., Molecular Gas Dynamics and the Direct Simulation of Gas Flows, New York: Oxford University Press, 1994.

[2] Rjasanow, S., and Wagner, W., Stochastic numerics for the Boltzmann equation, Springer, 2005.

[3] Bird, G., "Sophisticated DSMC," Notes prepared for a short course at the DSMC07 meeting, Santa Fe, USA, 2007.

[4] Boyd, I. D., "Analysis of rotational nonequilibrium in standing shock waves of nitrogen,” AIAA journal, Vol. 28, No. 11, 1990, pp. 1997-1999.

[5] Boyd, I., and Wang, W.-L., "Monte Carlo computations of hypersonic interacting flows," 39th Aerospace Sciences Meeting and Exhibit, 2001, p. 1029.

[6] Bondar, Y., Maruta, K., and Ivanov, M., "Detonation in Microchannel by the DSMC Method," 42nd AIAA Thermophysics Conference, 2011, p. 3129.

[7] Hadjiconstantinou, N. G., "Analysis of discretization in the direct simulation Monte Carlo," Physics of Fluids, Vol. 12, No. 10, 2000, pp. 2634-2638.

[8] Hadjiconstantinou, N. G., Garcia, A. L., Bazant, M. Z., and He, G., "Statistical error in particle simulations of hydrodynamic phenomena," Journal of computational physics, Vol. 187, No. 1, 2003, pp. 274-297.

[9] Jenny, P., Torrilhon, M., and Heinz, S., "A solution algorithm for the fluid dynamic equations based on a stochastic model for molecular motion,” Journal of computational physics, Vol. 229, No. 4, 2010, pp. 1077-1098.

[10] Gorji, M. H., Torrilhon, M., and Jenny, P., "Fokker-Planck model for computational studies of monatomic rarefied gas flows," Journal of fluid mechanics, Vol. 680, 2011, pp. 574-601.

[11] Mathiaud, J., and Mieussens, L., "A Fokker-Planck model of the Boltzmann equation with correct Prandtl number," Journal of Statistical Physics, Vol. 162, No. 2, 2016, pp. 397-414.

[12] Cercignani, C., “The boltzmann equation,” The Boltzmann Equation and Its Applications, Springer, 1988, pp. 40-103.

[13] Gorji, M. H., "Fokker-Planck solution Algorithm for rarefied gas flows and applications of complex gas-surface interactions," Ph.D. thesis, ETH Zurich, 2014.

[14] Risken, H., "Fokker-planck equation,” The Fokker-Planck Equation, Springer, 1996, pp. 63-95.

[15] Gorji, M. H., and Jenny, P., "A Fokker-Planck based kinetic model for diatomic rarefied gas flows," Physics of fluids, Vol. 25, No. 6, 2013, p. 062002.

[16] Bhatnagar, P. L., Gross, E. P., and Krook, M., "A model for collision processes in gases. I. Small amplitude processes in charged and neutral one-component systems," Physical review, Vol. 94, No. 3, 1954, p. 511. 
[17] Mathiaud, J., and Mieussens, L., "A Fokker-Planck Model of the Boltzmann Equation with Correct Prandtl Number for Polyatomic Gases," Journal of Statistical Physics, Vol. 168, No. 5, 2017, pp. 1031-1055.

[18] Øksendal, B., “Stochastic differential equations," Stochastic differential equations, Springer, 2003, pp. 65-84.

[19] Jiang, Y., Gao, Z., and Lee, C.-H., "Particle simulation of nonequilibrium gas flows based on ellipsoidal statistical Fokker-Planck model," Computers \& Fluids, Vol. 170, 2018, pp. 106-120.

[20] Gorji, M. H., and Jenny, P., “An efficient particle Fokker-Planck algorithm for rarefied gas flows,” Journal of Computational Physics, Vol. 262, 2014, pp. 325-343.

[21] Pfeiffer, M., and Gorji, M. H., "Adaptive particle-cell algorithm for Fokker-Planck based rarefied gas flow simulations," Computer Physics Communications, Vol. 213, 2017, pp. 1-8.

[22] Plimpton, S., and Gallis, M., "SPARTA Direct Simulation Monte Carlo (DSMC) Simulator," Sandia National Laboratories, USA, see http://sparta. sandia. gov.

[23] Jun, E., Boyd, I., and Burt, J., “Assessment of an All-Particle Hybrid Method for Hypersonic Rarefied Flow,” 51st AIAA Aerospace Sciences Meeting including the New Horizons Forum and Aerospace Exposition, AIAA paper 2013-1203, ????

[24] Küchlin, S., and Jenny, P., "Automatic mesh refinement and parallel load balancing for Fokker-Planck-DSMC algorithm," Journal of Computational Physics, Vol. 363, 2018, pp. 140-157.

[25] Gorji, M. H., and Torrilhon, M., “A Fokker-Planck model of hard sphere gases based on H-theorem,” AIP Conference Proceedings, Vol. 1786, 2016, p. 090001.

[26] Gorji, M. H., and Jenny, P., "Fokker-Planck-DSMC algorithm for simulations of rarefied gas flows," Journal of Computational Physics, Vol. 287, 2015, pp. 110-129. 


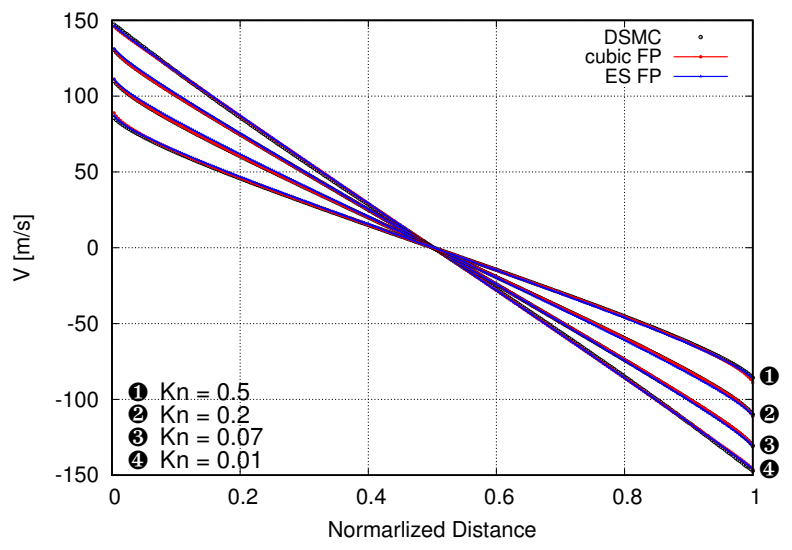

Figure 1 Mean velocity in Couette flow. Comparison is made between DSMC, cubic-FP and ES-FP results for $\mathbf{K n} \in\{0.01,0.07,0.2,0.5\}$.

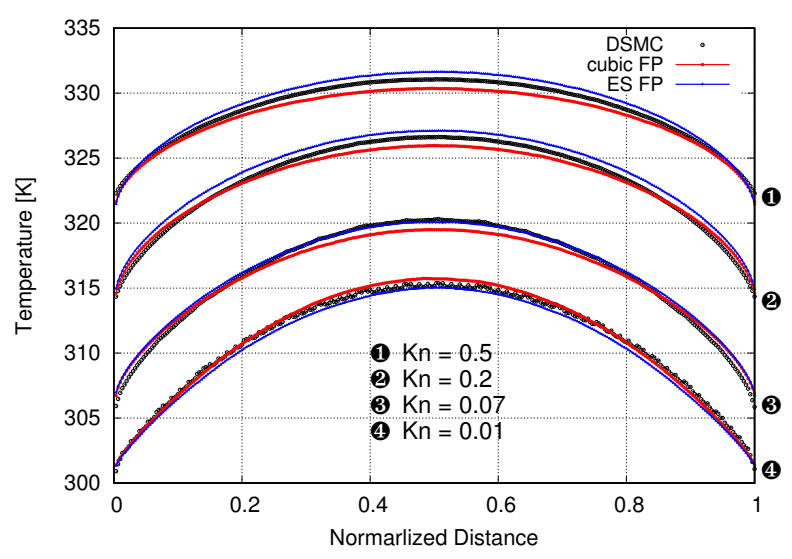

(a)

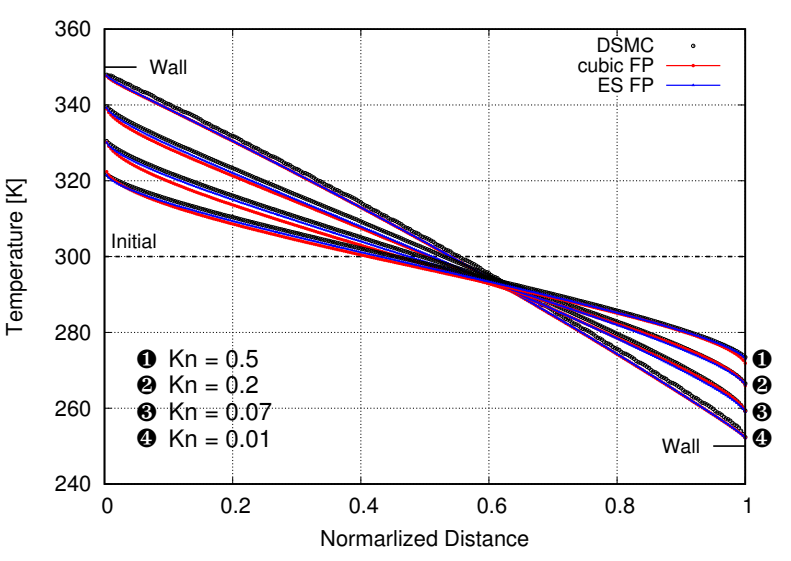

(b)

Figure 2 Temperature in (a) Couette flow (b) Fourier flow. Comparison is made between DSMC, cubic-FP and ES-FP results for $\mathrm{Kn} \in\{0.01,0.07,0.2,0.5\}$. 


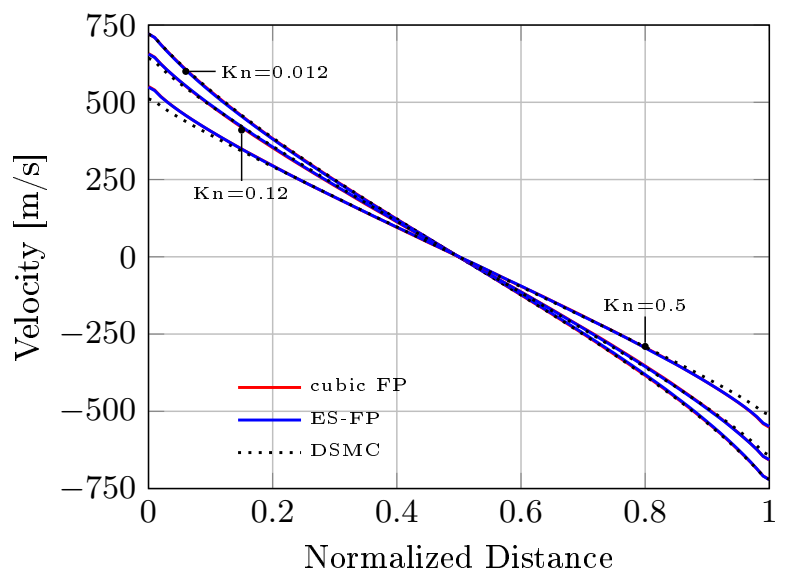

Figure 3 Velocity profiles of supersonic Couette flow. Comparison is made between DSMC, cubic-FP and ES-FP results for $\mathrm{Kn} \in\{0.012,0.12,0.5\}$.

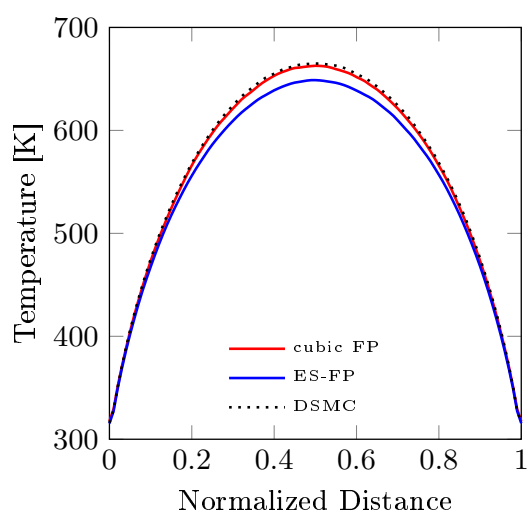

(a)

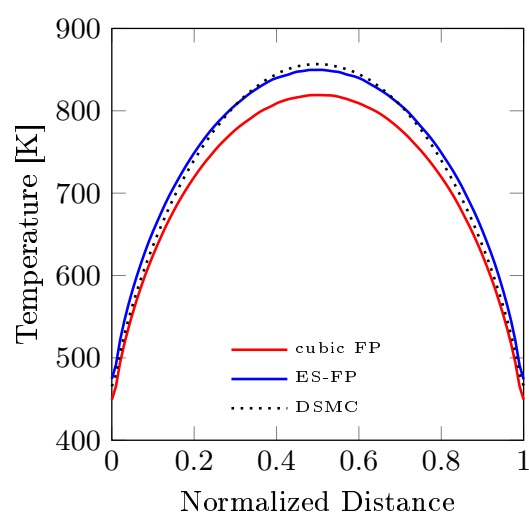

(b)

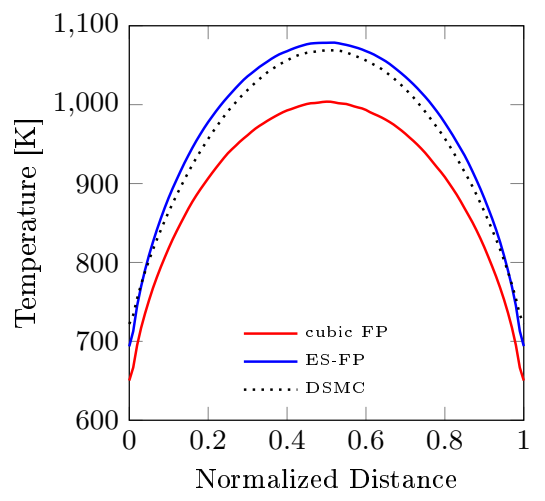

(c)

Figure 4 Temperature profiles of supersonic Couette flow ((a) $K n \approx 0.012$, (b) $K n \approx 0.12 \&$ (c) $K n \approx 0.5)$ using DSMC, cubic-FP and ES-FP.

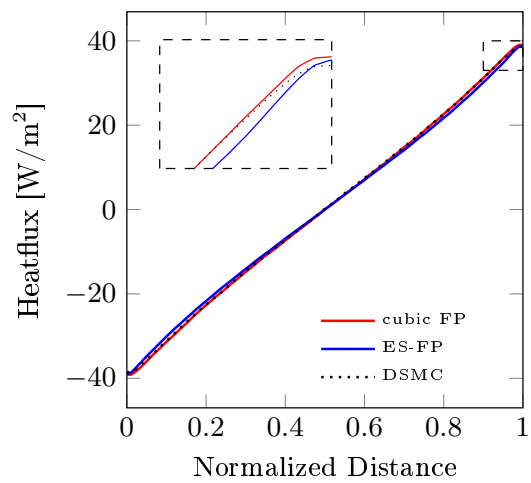

(a)

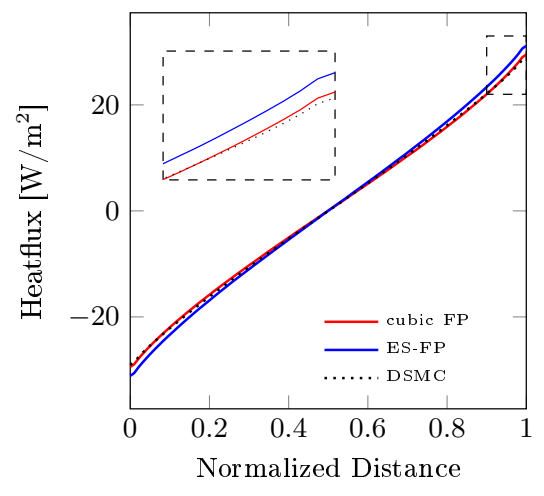

(b)

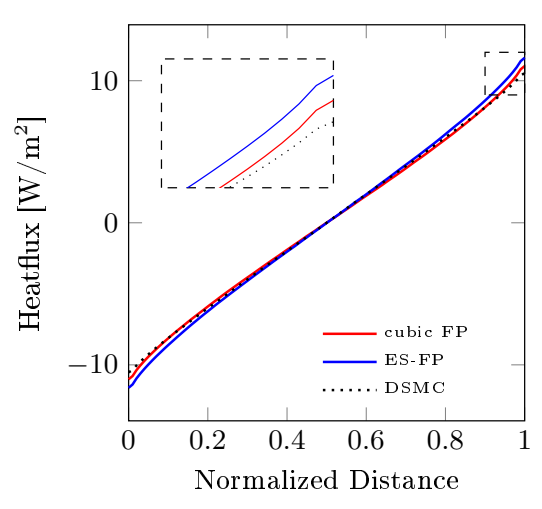

(c)

Figure 5 Heat-flux profiles normal to the wall (magnification of dashed regions) of supersonic Couette flow ((a) $K n \approx 0.012$, (b) $K n \approx 0.12 \&$ (c) $K n \approx 0.5)$ using DSMC, cubic-FP and ES-FP. 


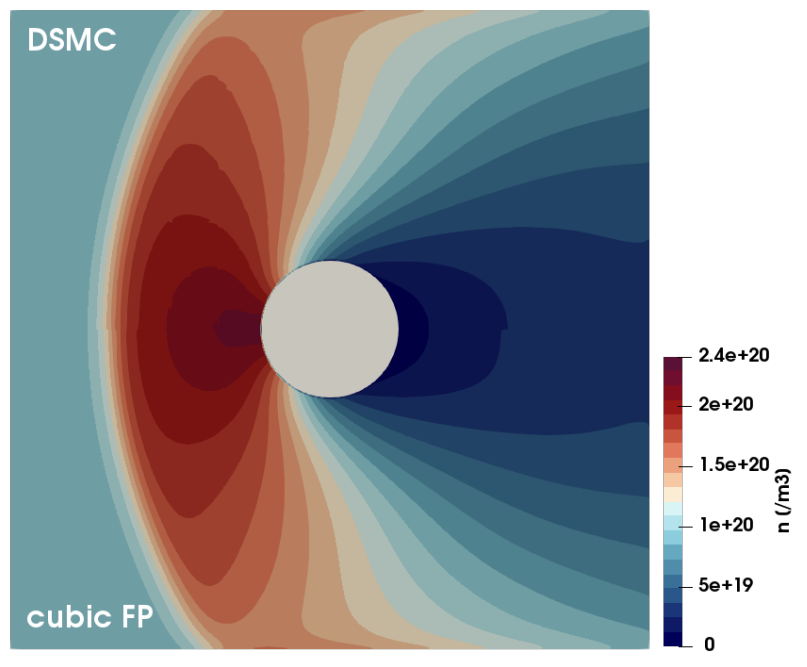

(a) Number density, $/ \mathrm{m}^{3}$

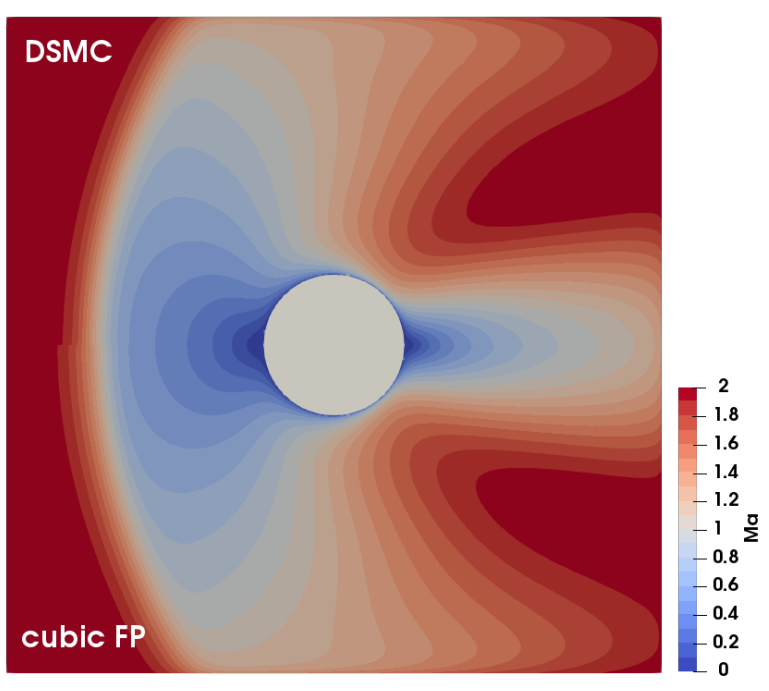

(c) Mach number

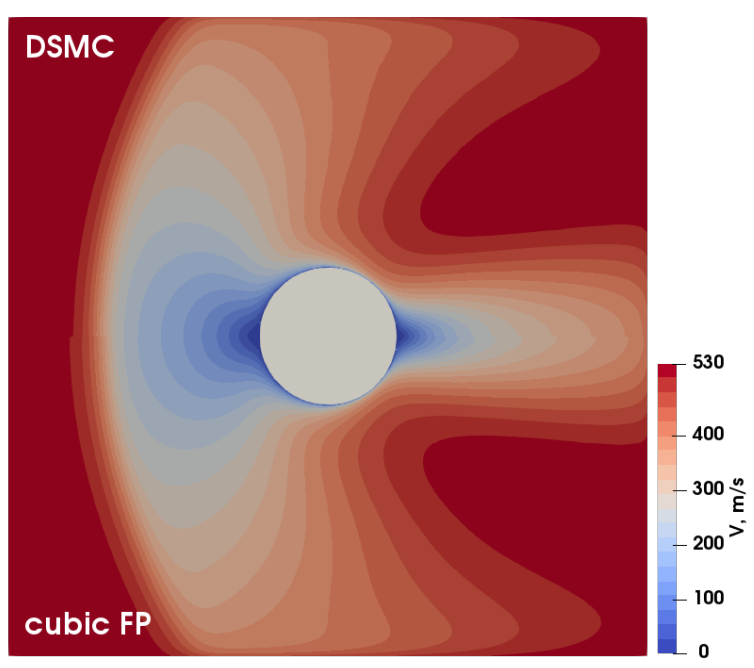

(b) Velocity, m/s

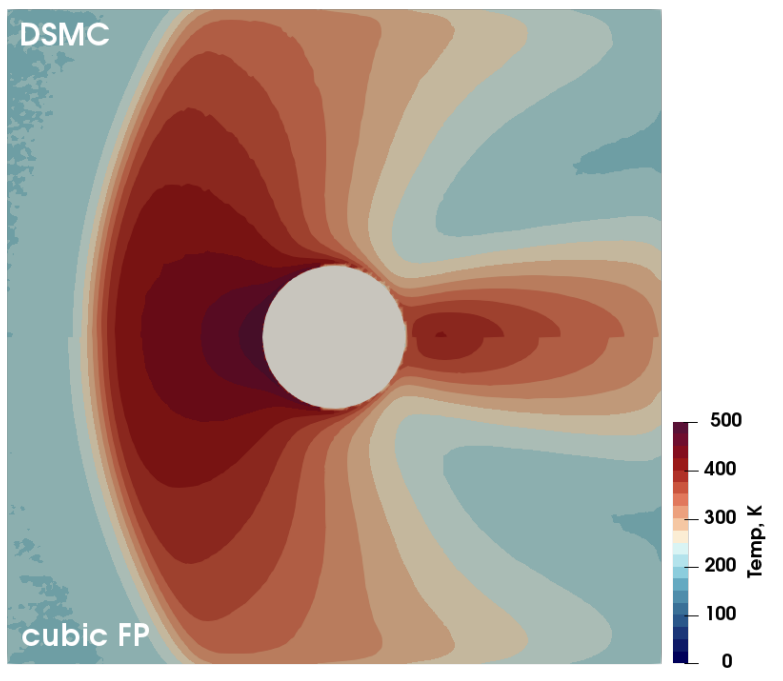

(d) Temperature, K

Figure 6 Mach and temperature contours for supersonic flow around the cylinder at $\mathrm{Kn}=0.05$ and $\mathrm{Ma}=2$, using DSMC and cubic-FP results. 


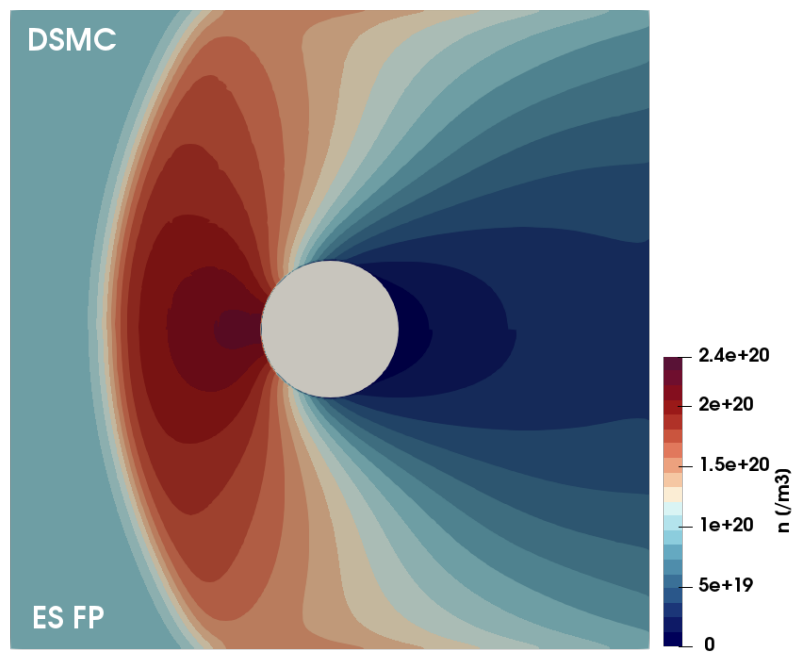

(a) Number density, $/ \mathrm{m}^{3}$

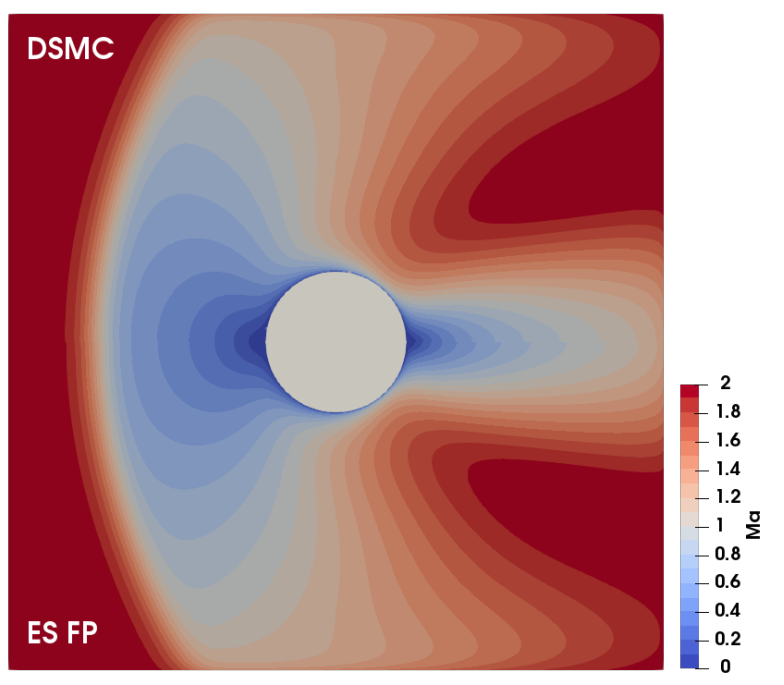

(c) Mach number

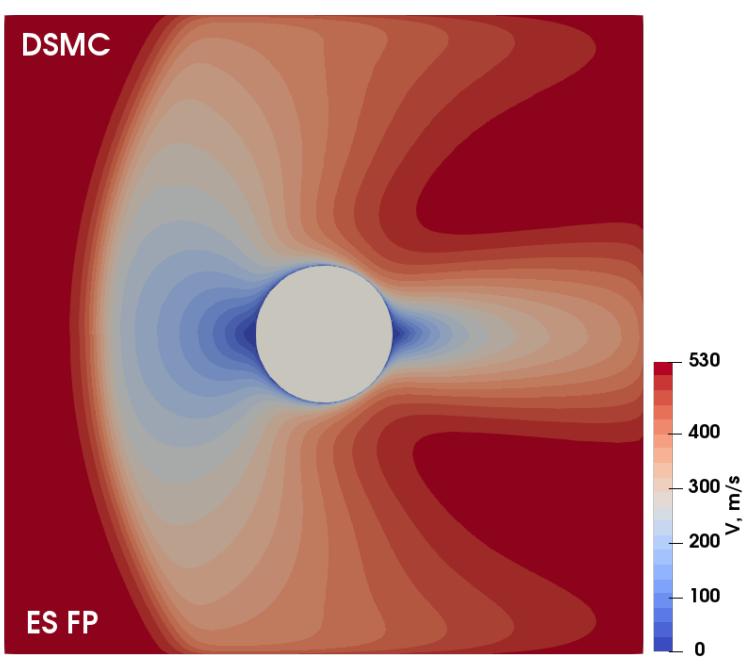

(b) Velocity, m/s

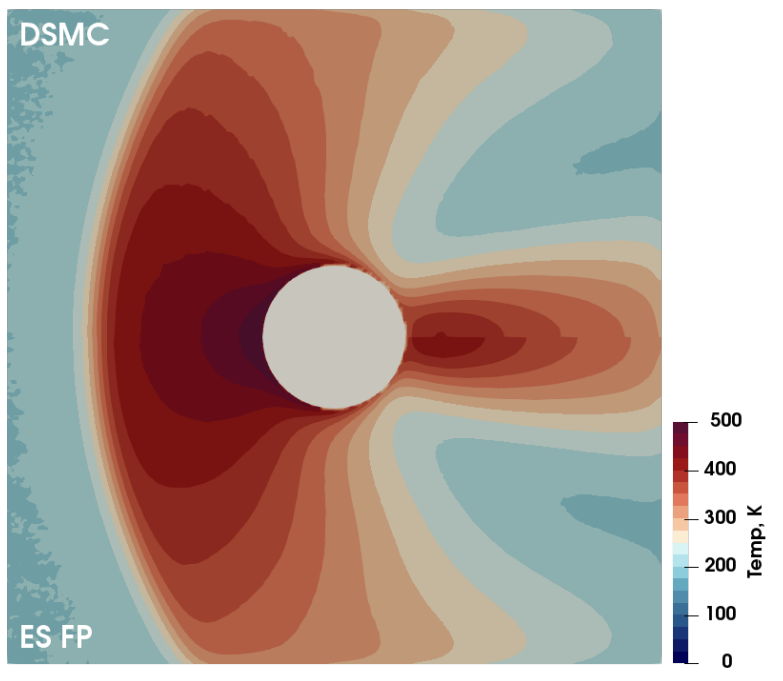

(d) Temperature, K

Figure 7 Mach and temperature contours for supersonic flow around the cylinder at $\mathrm{Kn}=0.05$ and $\mathrm{Ma}=2$, using DSMC and ES-FP results. 


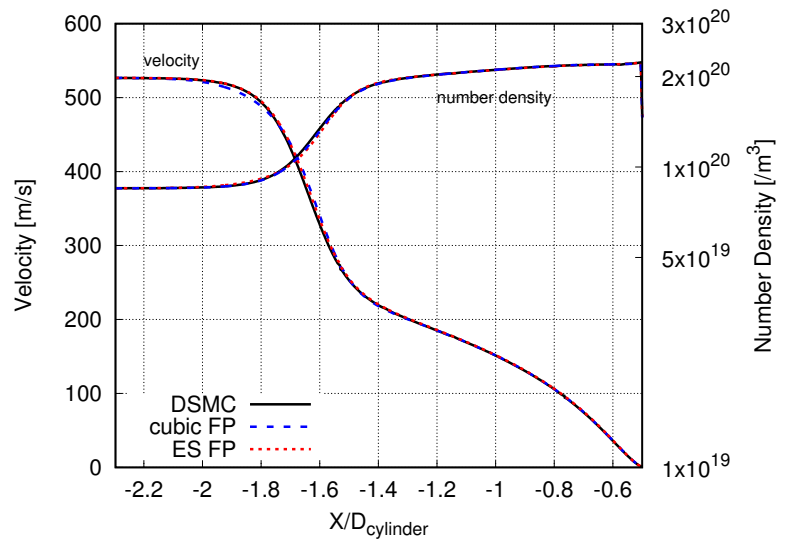

(a) Profiles of Velocity and number density along the stagnation line.

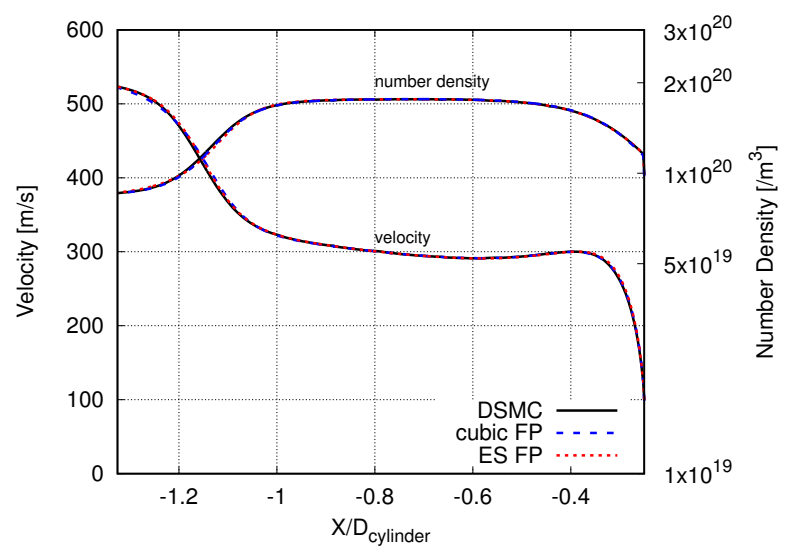

(c) Profiles of Velocity and number density along an extraction line inclined at $60^{\circ}$.

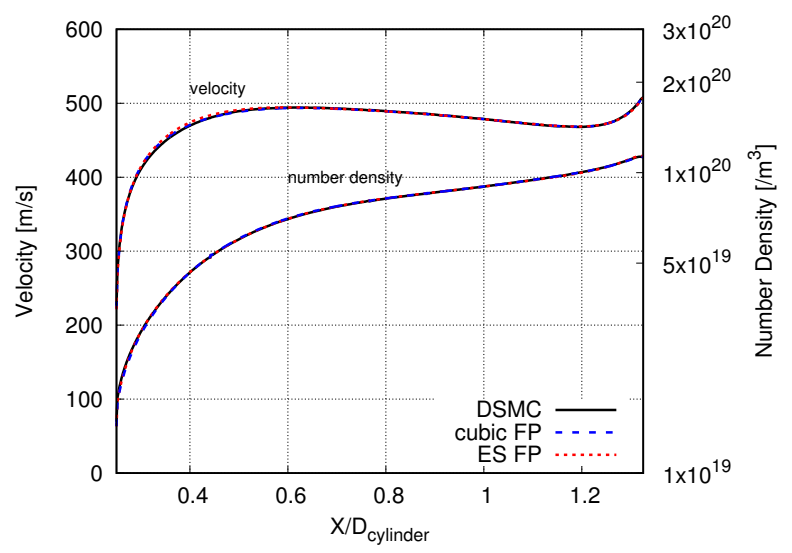

(e) Profiles of Velocity and number density along an extraction line inclined at $120^{\circ}$.

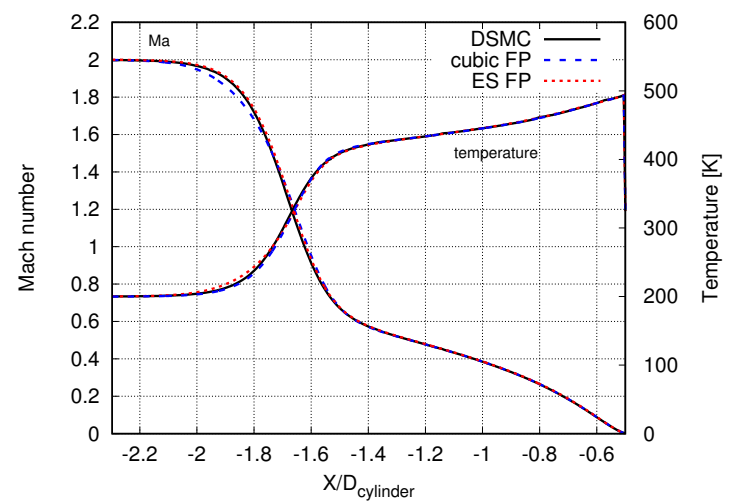

(b) Profiles of Mach number and temperature along the stagnation line.

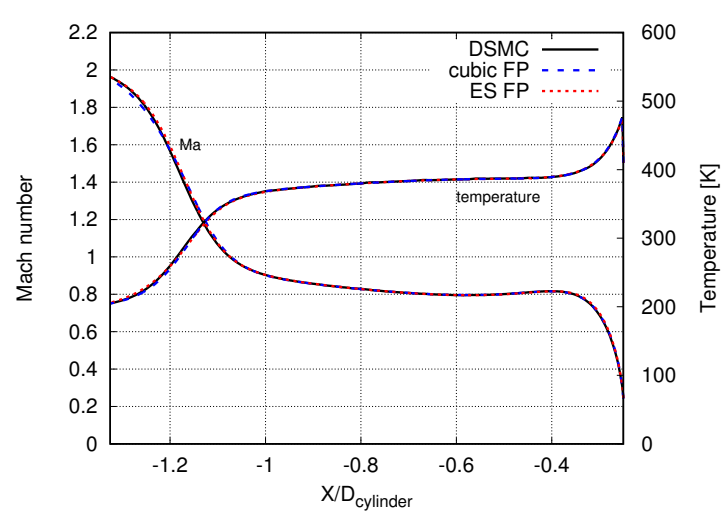

(d) Profiles of Mach number and temperature along an extraction line inclined at $60^{\circ}$.

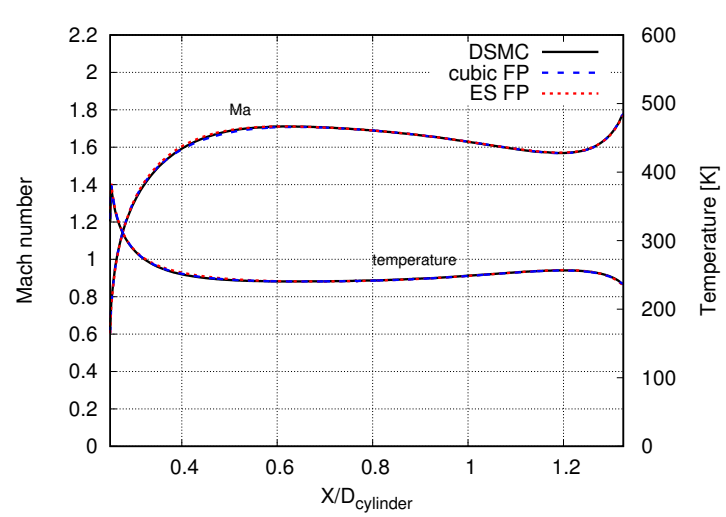

(f) Profiles of Mach number and temperature along an extraction line inclined at $120^{\circ}$.

Figure 8 Flow variables for supersonic flow around the cylinder using DSMC, cubic-FP and ES-FP results. 


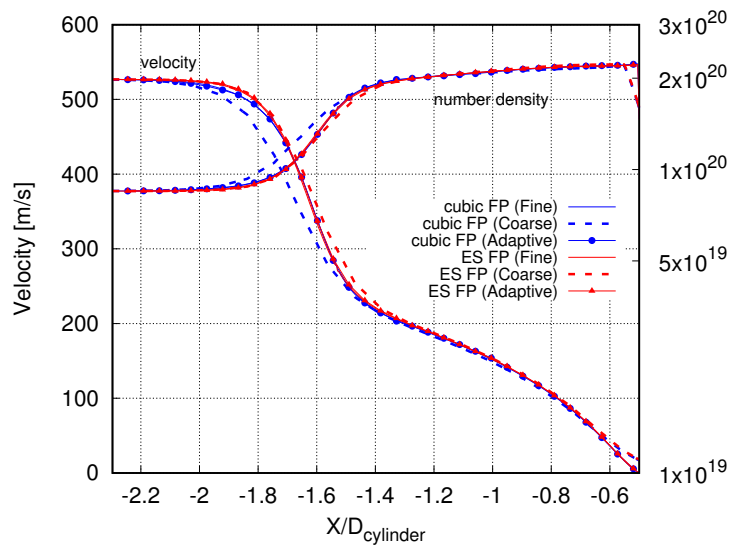

(a) Profiles of Velocity and number density along the stagnation line: Various grid configurations

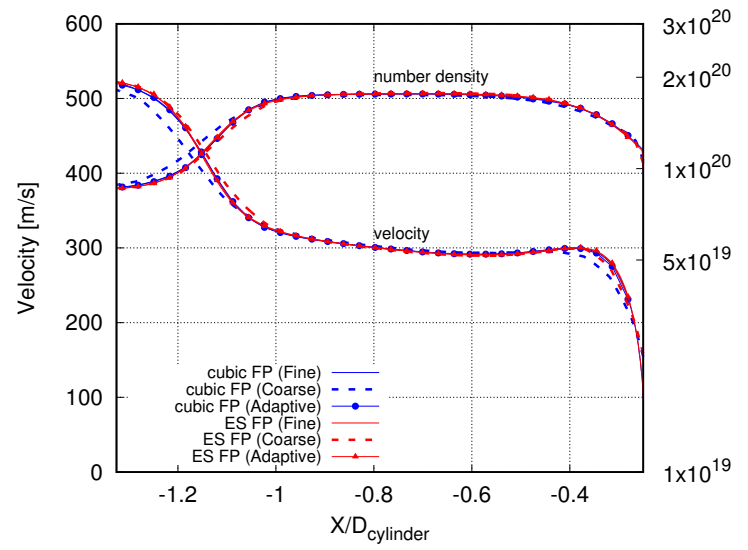

(c) Profiles of Velocity and number density along an extraction line inclined at $60^{\circ}$.

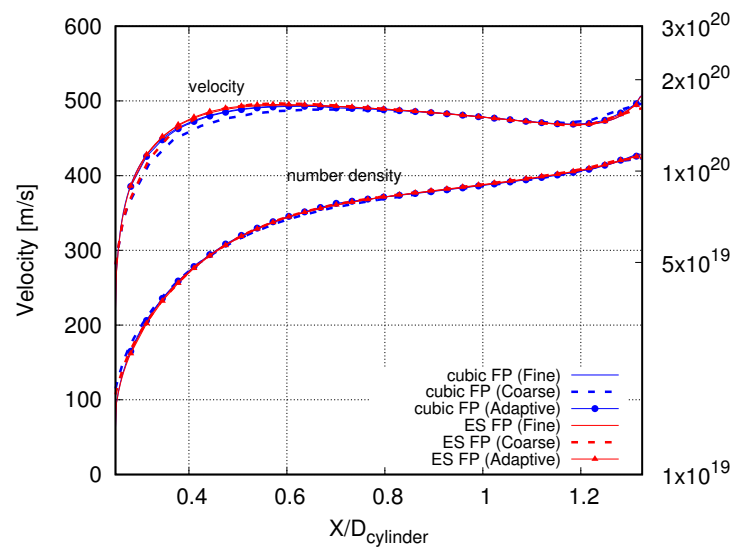

(e) Profiles of Velocity and number density along an extraction line inclined at $120^{\circ}$.

Figure 9 Flow variables for supersonic flow around the cylinder using DSMC, cubic-FP and ES-FP results.

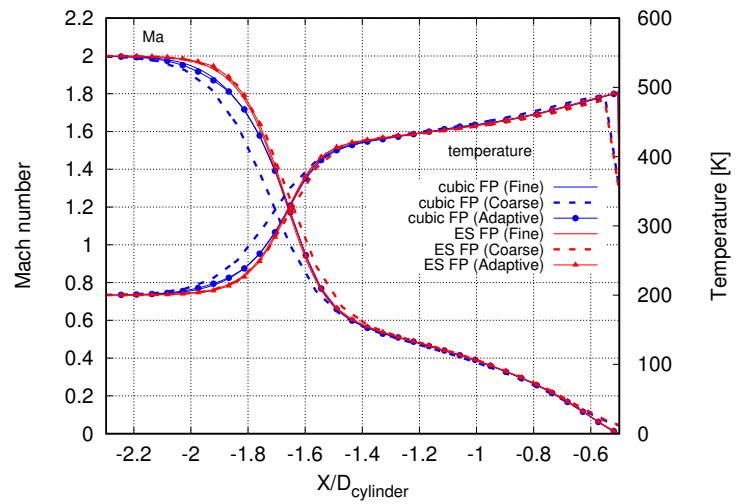

(b) Profiles of Mach number and temperature along the stagnation line.

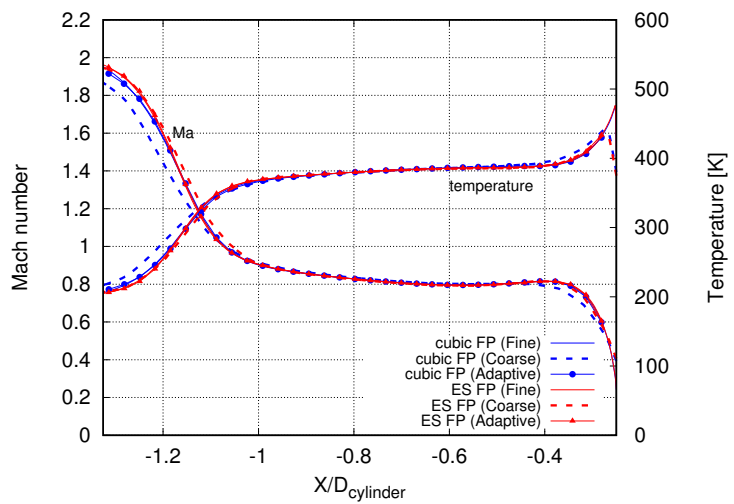

(d) Profiles of Mach number and temperature along an extraction line inclined at $60^{\circ}$.

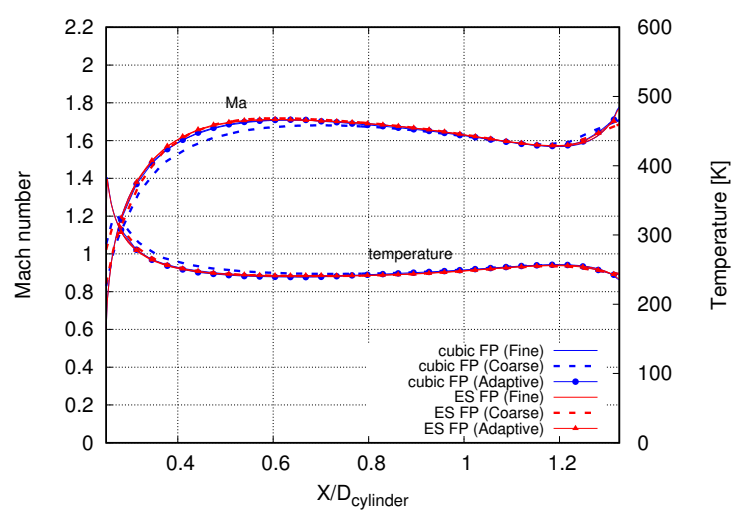

(f) Profiles of Mach number and temperature along an extraction line inclined at $120^{\circ}$ 


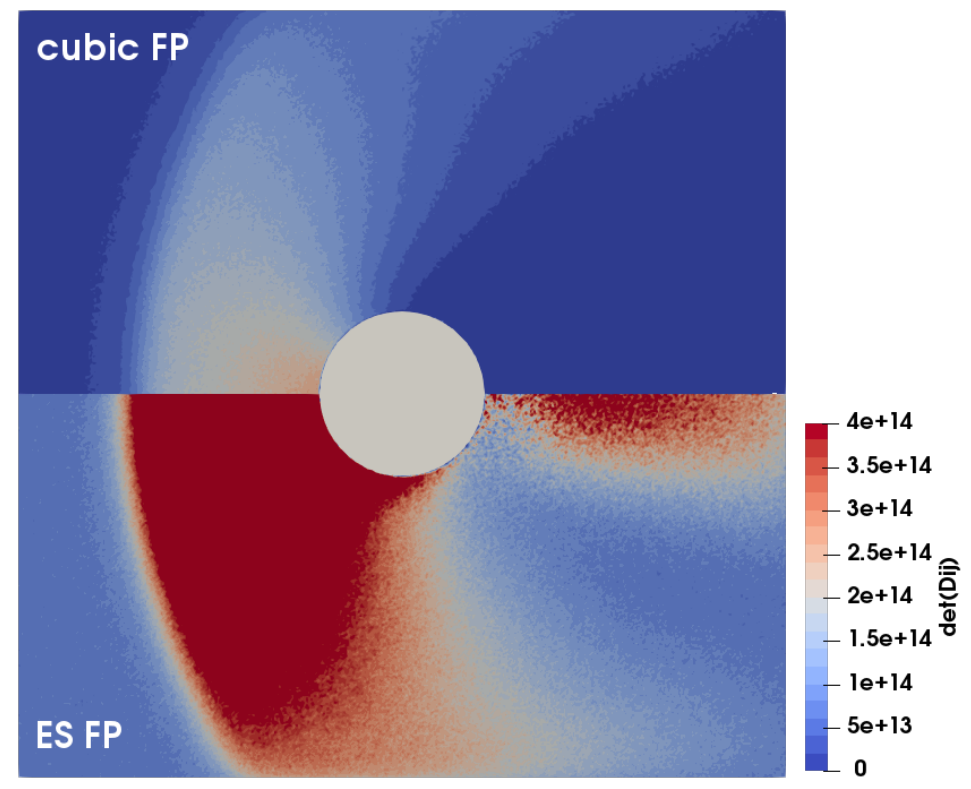

Figure 10 Determinant of the diffusion coefficient, $D_{i j}$ 